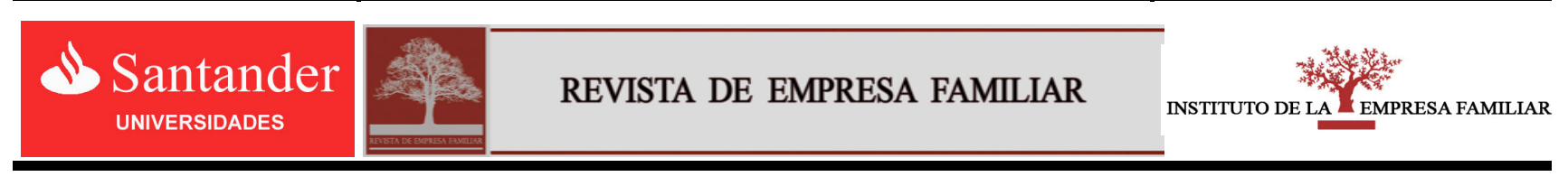

www.revistadeempresafamiliar.uma.es

\title{
Profesionalizarse, emprender y aliarse para que la empresa familiar continúe
}

\section{Professional management, entrepreneurship and alliances}

\author{
to the family firm continuity
}

Tomás M. Bañegil Palacios ${ }^{\mathrm{a},{ }^{*}} \cdot$ Ascensión Barroso Martínez ${ }^{\mathrm{b}} \cdot$ Juan Luis Tato Jiménez $^{\mathrm{c}}$

${ }^{a, b, c}$ Departamento de Dirección de Empresas y Sociología. Universidad de Extremadura, 06006 (Spain)

\begin{tabular}{l} 
D A T O S A R T Í C U L O \\
\hline Historial: \\
Recibido 03-11-2011 \\
Aceptado 23-11-2011 \\
Palabras clave: \\
Continuidad \\
Espíritu emprendedor \\
Gestión profesional \\
Alianzas y cooperación \\
Códigos JEL: \\
K36
\end{tabular}

A R T I C L E I N F O

Article history:

Received 3 November 2011

Accepted 23 November 2011

Keywords:

Continuity

Entrepreneurship

Professional management

Alliances and cooperation

JEL codes:

K36
RES UMEN

La continuidad de la empresa familiar es uno de los temas más estudiados. Los factores más utilizados en su estudio son la sucesión y la confusión entre el patrimonio empresarial y familiar. Sin embargo, en este trabajo vamos a analizar la continuidad de la empresa familiar y la influencia que sobre ella tienen algunas variables poco estudiadas; en concreto analizaremos la influencia que sobre ella tienen la realización de estrategias de alianzas y cooperación, la gestión profesional de la empresa familiar y el espíritu emprendedor de la empresa. La metodología utilizada para analizar estas relaciones está basada en un proceso de ecuaciones estructurales, concretamente el enfoque Partial Least Squares (PLS). Los resultados son, en la mayoría de las relaciones empleadas, los esperados según la literatura.

\section{A B S T R A C T}

Family firm continuity is one of the most studied issues. Succession and confusion between business and family heritage are the most used factors in its study. However, in this paper we analyze the family business continuity and the influence upon it of some poorly studied variables; actually we examine the influence of alliance and cooperation strategies, professional management and entrepreneurship on family firm continuity. The methodology used to analyze these relationships is based on a process of structural equations; in particular Partial Least Squares approach (PLS). The results are, in most of relationships, as expected from the literature.

Autor de contacto.

Correoselectrónicos: tbanegil@unex.es, abarrosom@unex.es, jltato@unex.es 


\section{Introducción}

La empresa familiar es una organización social de gran complejidad, ya que en ella conviven la empresa y la familia. Estos dos subsistemas no sólo se superponen sino que son interdependientes (Leach, 2002), lo cual genera una gran cantidad de conflictos que deben superar las empresas familiares si quieren sobrevivir y posicionarse de manera exitosa en el mercado. La continuidad de dichas empresas es un tema crítico debido a su peso dentro de la realidad socio-económica de cualquier país. De ahí que éste haya sido uno de los temas más que ha merecido mayor atención (Gallo y Amat, 2003).

Es frecuente que se destaquen sólo los aspectos problemáticos de las empresas familiares, tanto desde la perspectiva empresarial como personal. En este sentido, este trabajo hará mayor hincapié sobre las cuestiones que amenazan y favorecen la continuidad de la empresa familiar.

La interacción de los sistemas propiedad, empresa y familia nos conduce a dos problemas principales que afectan de manera significativa a la continuidad de este tipo de empresas. El primero es la confusión entre el patrimonio empresarial y el familiar y los conflictos que ello supone (Cole, 2000; Kellermanns y Eddleston, 2004). El segundo es la transmisión del poder y liderazgo de una generación a otra, es decir, la sucesión (Le Breton-Miller, Miller y Steier, 2004; Royer, Simons, Boyd y Rafferty, 2008).

Estos temas han sido tratados por numerosos autores e investigadores de la materia y todos llegan a un consenso identificando a la sucesión como una de las principales causas de mortandad en la mayoría de este tipo de negocios. Sobre estas cuestiones podrían destacarse los trabajos de Handler (1994); Dyck, Mauws, Starke y Mischke, 2002) y Cabrera y Martín (2010) entre otros.

Sin embargo, nuestra investigación aporta otros aspectos que también influyen en la continuidad de las empresas familiares y han sido menos estudiados, ya que los mencionados anteriormente han sido tratados en numerosos trabajos teóricos y empíricos.

Nuestro objetivo, por tanto, es analizar la continuidad de la empresa familiar y la influencia que sobre ella tienen algunas variables relacionadas con la gestión y la estrategia, factores poco estudiadas en su relación con la continuidad de la misma. En concreto analizaremos la influencia que sobre ella tienen la realización de estrategias de alianzas y cooperación, la gestión profesional de la empresa familiar y el espíritu emprendedor de la empresa. A su vez, analizamos la relación de estas variables entre sí.

El trabajo se estructura de la siguiente forma. La primera sección es de carácter introductorio. En la segunda sección se propone un modelo y una serie de hipótesis para contrastarlo; para ello se realiza una revisión de la literatura que proporciona una descripción de las relaciones entre espíritu emprendedor, gestión profesional de la empresa familiar, formación de alianzas y cooperación con la continuidad. La siguiente sección se dedica a la descripción de la metodología utilizada, seguida de la presentación de los resultados obtenidos. Finalmente se presentas las conclusiones principales del trabajo, las futuras líneas de investigación y las limitaciones del mismo.

\section{La continuidad en la empresa familiar}

Existe una coincidencia unánime por parte de los expertos al señalar que el principal inconveniente de las empresas familiares radica en la dificultad de mantener la continuidad a lo largo de distintas generaciones. Por tanto, es una cuestión de importancia esencial conseguir una adecuación entre el ciclo vital de la empresa y la gestión óptima en cada ciclo. A medida que transcurre el tiempo y se transforman las relaciones familiares, es posible que sea necesario modificar el estilo y las claves de gestión dado que aquello que funcionaba en un determinado momento del ciclo de vida, puede ser manifiestamente mejorable en otro momento.

El promedio de vida de las empresas familiares es preocupante y las razones que

Bañegil Palacios, T.M., Barroso Martínez, A. y Tato Jiménez, J.L. (2011). Profesionalizarse, emprender y aliarse para que la empresa familiar continúe. Revista de Empresa Familiar, 1(2), 27-41 
explican la mortalidad estimulan su investigación. En término medio, tasa de desaparición de empresas familiares es bastante elevada: un $65 \%$ de las empresas familiares se encuentra en primera generación; un $25 \%$, en segunda; un $9 \%$, en tercera y tan sólo un $1 \%$ en cuarta o ulterior generación (Gallo, 1998), por lo que resulta imprescindible profundizar en sus causas. Entre los principales causantes de la no continuidad de las empresas familiares se encuentran la falta de emprendedurismo, la falta de gestión profesional (Fernández y Bringmann, 2007) y la falta de iniciativa para realizar estrategias de alianzas y cooperación (Claver, Rienda y Pertusa, 2004). Ya que en una organización la existencia de una determinada línea estratégica que haya conseguido el éxito en el pasado y la falta de flexibilidad pueden convertirse en inconvenientes si se produce resistencia a las innovaciones, sobre todo si la empresa se encuentra escasamente profesionalizada.

\subsection{La continuidad y la gestión profesional en la empresa familiar}

En los últimos años se viene debatiendo bastante a cerca de la imprescindible profesionalización de la gestión en este tipo de organizaciones. Profesionalizar el manejo de la empresa es dejar de lado los métodos intuitivos de gestión familiar (McCann, Leon-Guerrero y Haley, 2001), y adoptar la planificación y el control del crecimiento, aplicando métodos estratégicos de gestión (Miller, McLeod y Oh, 2001).

Esto implica el desarrollo de un plan de negocio, fijando una estrategia en función de las características de los mercados donde queramos operar, las políticas para poner en marcha la actividad, la rentabilidad económica que podamos alcanzar y los planes de contingencia con que abordar cambios de los escenarios previamente establecidos.

Para poder conseguir todo esto es necesario gestionar eficazmente los sistemas familia $\mathrm{y}$ empresa (Chu, 2011) (siendo este uno de los principales problemas en la continuidad de las empresas familiares) mediante la adopción de estructuras y políticas de gobierno que exijan colaboración y poder compartido (Lansberg, 2007); es decir, a través de los órganos de gobierno, tanto exclusivos como no exclusivos de la empresa familiar (Jaskiewicz y Klein, 2007).

Para lograr un equilibrio entre la empresa, la propiedad y la familia, la empresa ha de contar con una junta general y con un consejo de administración eficaz, instituciones típicamente empresariales que han de adaptarse a las singularidades de este tipo de organizaciones para poder cumplir con mayor eficacia las funciones que tienen encomendadas (SánchezCrespo, Bellver y Sánchez, 2005). A principios de la década de los noventa las empresas familiares comenzaron a reconocer la necesidad de complementar el gobierno de la empresa con el gobierno de la familia (Martínez, 2007); constatándose así la necesidad de instrumentos que permitan un mejor funcionamiento de su sistema de valores. De este modo se crearon la Asamblea Familiar y el Consejo de Familia, entre otros. Estos órganos de gobierno no están previstos en la legislación societaria, pero pueden incorporarse en los Estatutos Sociales siempre que no invadan o limiten las competencias de los órganos sociales necesarios (Gortázar, 2007).

La Asamblea Familiar es un órgano de carácter informativo y no decisorio que aglutina a todos los miembros de la familia, a partir de una cierta edad, trabajen o no en la empresa y sean o no propietarios de la misma (Galve, 2002). A partir de la tercera generación es conveniente crear el Consejo de Familia, que es un órgano de carácter decisorio que regula su funcionamiento y sus relaciones con la empresa, y en cuyo seno se discuten tanto los problemas del presente como proyectos de futuro de la familia empresaria. Este órgano contribuye a fortalecer y mantener activos los valores y la historia de la familia, preservando su unidad y armonía (Blumentritt, Keyt y Astrachan, 2007).

Por tanto, conforme la empresa familiar madura y van surgiendo formas organizacionales más complejas, el solapamiento entre la familia y la empresa genera conflictos en la organización. La 
solución a estos conflictos requiere la implantación y adopción de ciertos instrumentos y órganos de gobierno exclusivos de la empresa familiar y de la familia empresaria. Los órganos de gobierno han de servir para dirigir y encauzar las relaciones familia-empresa con el fin de garantizar la continuidad de la empresa familiar.

Pero además, cuando hablamos de profesionalización en la empresa familiar nos estamos refiriendo también a la contratación de profesionales ajenos a la familia (Giovannoni, Maraghini y Riccaboni, 2011) para ocupar puestos claves en la toma de decisiones (Blumentritt, Keyt y Astrachan, 2007). No se trata de evitar que los puestos directivos sean ocupados por miembros de la familia, pero sí de que un miembro de la familia empresaria, por el mero hecho de serlo, ocupe un puesto directivo sin poseer las competencias adecuadas para ello.

Cada puesto debe ser desempeñado por el mejor candidato posible, sea éste un miembro familiar o no (Hall y Norqvist, 2008). Por tanto, no debería ser un objetivo prioritario de la organización encontrar un puesto de trabajo en la empresa a los familiares (Chittoor y Das, 2007). Por ello es necesario que los miembros de la familia que dirijan la organización comprendan detalladamente la empresa, estén capacitados y apliquen métodos profesionales de gestión (Ibrahim, Soufani, Poutziouris y Lam, 2004). Además, es deseable que tengan experiencias previas en otras organizaciones antes de ingresar en la empresa ya que les otorgarán una invalorable formación que la empresa familiar podrá capitalizar (Chirico, 2008).

Profesionalizar y potenciar los talentos en las empresas familiares es un proceso complejo que no solo se resuelve con miembros de la familia capacitados, o con la incorporación de ejecutivos. Va mucho más allá, dado que implica un cambio en la cultura de la empresa hacia una dirección más profesional. Como señala Leach (2002), es un paso decisivo para manejar los conflictos potenciales entre los valores y metas de la familia y de la empresa. En esta línea de actuación, nuestra primera hipótesis es la siguiente:
$\mathrm{H}_{1}$ : La gestión profesional de la empresa familiar influye positivamente en la continuidad del negocio.

\subsection{La continuidad y los factores que influyen en la realización de estrategias de alianzas y cooperación en la empresa familiar}

Otro de los aspectos que vamos a tratar y con el que pueden contar las empresas familiares para enfrentarse a la globalización y así a la continuidad del negocio es la realización de alianzas estratégicas y acuerdos de cooperación (Fernández y Nieto, 2005; Ulubasoglu, Akdis y Kök, 2009). Muchas de las empresas familiares, aunque son fuertes en bastantes aspectos tienen carencias en ciertos recursos y capacidades, por lo que requieren de acuerdos de cooperación para llegar a otros mercados (Lozano, 2003) y crecer (Fernández, 1996). Además, son un medio eficaz para el proceso de internacionalización, pues les permiten compartir y encontrar recursos financieros, tecnológicos, humanos, organizativos $\mathrm{y}$ relacionales de las otras empresas socias. De hecho, los acuerdos de cooperación son una vía para mejorar el rendimiento internacional de las empresas familiares (Fernández y Nieto, 2005).

Las características peculiares que presentan estas empresas (Tagiuri y Davis, 1996) pueden influir notablemente en la formación y desarrollo de alianzas estratégicas.

La autonomía financiera (James, 1999) y la autonomía en la toma de decisiones (Donckels y Lambrech, 1999) dificultan la formación de alianzas. Normalmente los propietarios de las empresas familiares se muestran reacios a la entrada de capital externo como alternativa de financiación (Sánchez-Crespo, 2009), ya que no están dispuestos e incluso temen perder el control de la empresa. Ambas circunstancias podrían afectar tanto a la elección del compañero para formar la alianza como a la forma jurídica de la misma, e incluso podría poner en peligro la continuidad de la empresa familiar. Así, Swinth y Vinton (1993) argumentan que las alianzas estratégicas entre empresas familiares tienen mayores probabilidades de éxito debido a que ambas 
comparten determinados objetivos, valores y convicciones. Por ello también nos podemos encontrar con ciertas características de las empresas familiares que favorecen la formación de alianzas y cooperación, como son la flexibilidad y rapidez en la toma de decisiones, la orientación a largo plazo y los mayores niveles de compromiso, entre otros (Roessl, 2005; Claver, Rienda y Quer, 2008; Fuentes y Fernández, 2008).

En suma, Fuentes, Vallejo y Fernández (2011) identifican tres aspectos claves que pueden influir en la formación y desarrollo de acuerdos de cooperación: (1) que la alianza implique la participación de la empresa socio en el capital de la empresa familiar; (2) que la empresa socio sea también una empresa familiar y (3) que la empresa presente algunas de las debilidades que caracterizan a la empresa familiar.

Por tanto, y siguiendo con estos autores, las alianzas estratégicas ofrecen la posibilidad a las empresas familiares de mejorar su posición competitiva (Van Gils y Zwart, 2009) e incrementar sus recursos y capacidades, pudiendo garantizar beneficios a largo plazo, lo que supondría asegurar la continuidad de la empresa familiar a las siguientes generaciones. De este modo, nuestra siguiente hipótesis de estudio propuesta es:

$\mathrm{H}_{2}$ : La formación de alianzas y cooperación influye en la continuidad del negocio.

La falta de confianza hacia otras empresas para aliarse podría solventarse profesionalizando la empresa familiar, es decir, mediante la contratación de directivos profesionales no pertenecientes a la familia que aporten su experiencia y formación en el área concreta que se desea potenciar (Gallo, Ariño, Máñez y Cappuyns, 2004) . De este modo, los profesionales externos mejoran las aspiraciones y confianza del empresario; ayudan a perfilar y mejorar la visión del negocio y potencian el nivel de compromiso; permiten detectar las debilidades y fortalezas de las decisiones antes de que estas tengan repercusión en el negocio, etc. La necesidad de profesionalizar la gestión, aparece como un imperativo y una cuestión esencial para la supervivencia de las empresas familiares en escenarios inciertos y globalizados como los que actualmente vivimos. A partir de ahí formulamos la siguiente hipótesis:

$\mathrm{H}_{3}$ : La gestión profesional de la empresa familiar influye positivamente en la formación de alianzas y cooperación

Otro aspecto que afecta a la formación de una alianza es la presencia de determinados valores compartidos por los miembros de la familia, en este caso nos referimos a la asunción de riesgos o de periodos de tiempo fijados para la recuperación de inversiones y a una visión y actitud menos conservadora y más arriesgadas (Gallo, Ariño Máñez y Cappuyns, 2004; Fuentes, Vallejo y Fernández, 2011). Proponemos como hipótesis la siguiente:

$\mathrm{H}_{4}$ : El espíritu emprendedor influye positivamente en la formación de alianzas y cooperación.

\subsection{La continuidad y el espiritu emprendedor en la empresa familiar}

La nueva economía en la que nos encontramos se caracteriza por la globalización de los mercados, los avances en las tecnologías de la información y de la comunicación, las nuevas industrias emergentes y los rápidos cambios en la demanda de los consumidores. Para estar al día con estos cambios que afectan a muchas empresas y poder dar continuidad a los negocios, se hace necesario fomentar el espíritu emprendedor de las empresas (Hall, Melin, Nordqvist, 2001). Siguiendo con estas ideas, estamos convencidos del papel esencial que juegan las empresas familiares.

Un aspecto crucial del emprendedurismo implica el reconocimiento de oportunidades de negocios emergentes, las cuales son a menudo explotadas a través de las creación de nuevas empresas (Aldrich y Cliff, 2003). Así, el espíritu emprendedor es un concepto útil que conduce a las empresas en cómo participan en el cambio y en la renovación de procesos para mantener y mejorar su competitividad y continuidad. A partir de estas argumentaciones proponemos como hipótesis: 
$\mathrm{H}_{5}$ : El espíritu emprendedor influye positivamente en la continuidad del negocio.

Hay una gran diversidad de definiciones de espíritu emprendedor que podemos encontrar en la literatura existente, las cuales han enfatizado algunas actividades como la creación de nuevas organizaciones, la innovación, la asunción de riesgos, la nueva combinación de factores existentes, la exploración y explotación de oportunidades, la asunción de incertidumbre, la unión de los factores de producción, etc. (Ulhøi, 2005; Shane y Venkataraman, 2000; Audretsch, Bönte y Keilbach, 2008; Pistrui, Huang, Oksoy, Jing y Welsch, 2001).

Estudios relacionados con empresas familiares y el espíritu emprendedor muestran que una fuerte cultura empresarial relacionada con la familia podría impactar sobre la habilidad para crear y mantener capacidades emprendedoras en la empresa familiar (Casillas, Moreno y Barbero, 2010). Por tanto, por un lado nos podemos encontrar con empresas familiares innovadoras, proactivas y emprendedoras; y por otro lado, nos podemos encontrar con empresas familiares conservadoras, tradicionales, introvertidas y adversas al riesgo (Naldi, Nordqvist, Sjöberg y Wiklund, 2007). De este modo, creemos que las empresas familiares más emprendedoras y con una cultura más abierta tendrán una gestión más profesional en su empresa. Por ello, planteamos la siguiente hipótesis del estudio:

$\mathrm{H}_{6}$ : El espíritu emprendedor influye positivamente en la gestión profesional de la empresa familiar.

Finalmente, las hipótesis planteadas en este capítulo se plasman en el modelo conceptual propuesto, el cual analiza aspectos que podrían influir en la continuidad de las empresas familiares. Este modelo, reflejado en la figura 1, asume que la continuidad de una empresa familiar está influenciada por el espíritu emprendedor, la gestión profesional y los factores que influyen a la hora de formar alianzas y acuerdos de cooperación.

\section{Metodología}

La descripción de la metodología empleada se estructura en cinco partes: definición de la población y de la muestra, diseño y elaboración del cuestionario, trabajo de campo, modelo de medida y evaluación del modelo de investigación.

\subsection{Definición de la población y de la muestra}

La población de estudio se enmarca entre las

\section{Figura 1}

Modelo conceptual.

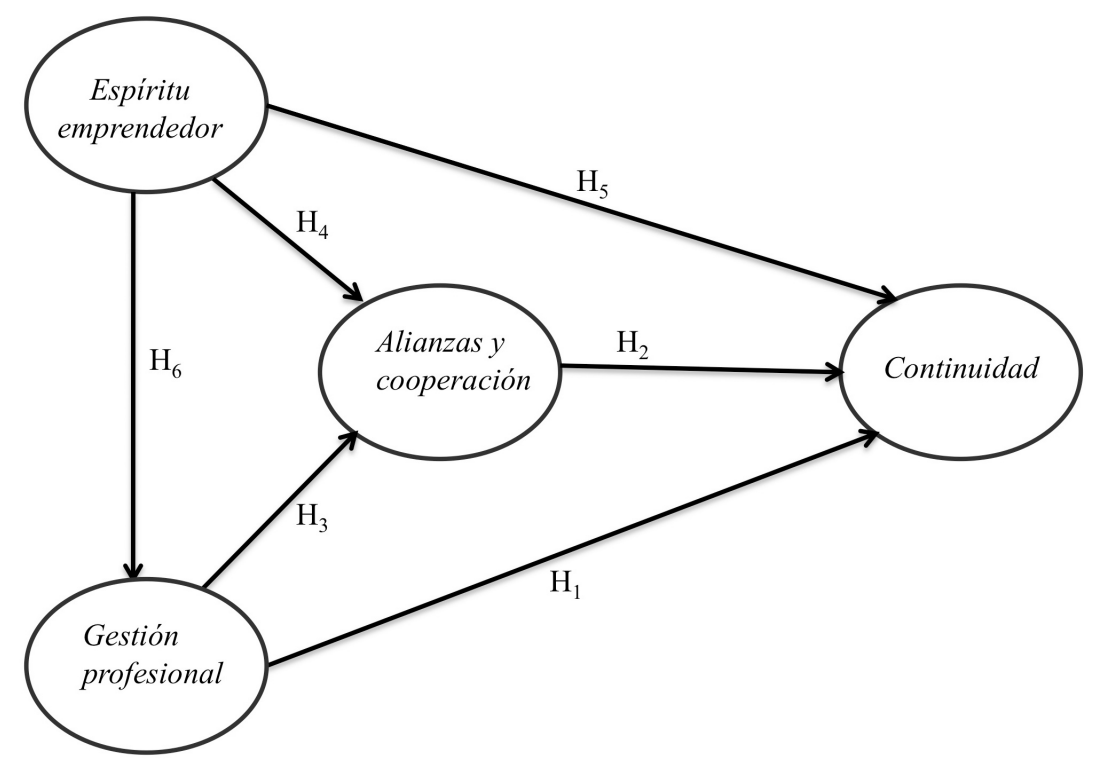

Fuente: elaboración propia

Bañegil Palacios, T.M., Barroso Martínez, A. y Tato Jiménez, J.L. (2011). Profesionalizarse, emprender y aliarse para que la empresa familiar continúe. Revista de Empresa Familiar, 1(2), 27-41. 
empresas que tienen domicilio social en la Comunidad Autónoma de Extremadura, por ello, metodológicamente, nuestro primer objetivo fue establecer una base de datos para conocer el porcentaje de las empresas familiares extremeñas. Así, en primer lugar se seleccionó una muestra de empresas extremeñas a partir de la base de datos ARDAN, incluida en la Guía Empresarial de Extremadura de 2008.

Puesto que el número de empresas incluidas en ARDAN es muy elevado, nos vimos obligados a introducir una restricción, utilizar solo las empresas con más de 5 empleados. Así, muestra población objeto de estudio estaría formada por 3.767 empresas. Para averiguar cuáles de estas empresas son familiares realizamos un cuestionario inicial, obteniendo que más del $70 \%$ de las empresas extremeñas son familiares. Para conocer más afondo las características de estas empresas realizamos 180 entrevistas personales.

\subsection{Diseño y elaboración del cuestionario}

El cuestionario inicial nacía con el propósito de ser utilizado como test inicial para identificar las empresas familiares existentes en Extremadura. El cuestionario final (Anexo 1) se elaboró a partir de la literatura existente y de la realización de un Delphi, cuyos expertos pertenecen a la Red de Cátedra de Empresa Familiar.

\subsection{Trabajo de campo}

En primer lugar se envió una carta de presentación a las empresas familiares que estaban dispuestas a seguir colaborando con nosotros junto con el propio cuestionario. Después se llamó a dichas empresas para concertar una cita en su localidad para cumplimentar el cuestionario. La aplicación del mismo se desarrolló a través de entrevistas personales en la empresa del informante clave.

\section{Modelo de medida, evaluación y contrastación de hipótesis}

\subsection{Modelo de medida}

Para el análisis de los datos hemos utilizado

\section{Figura 2}

Modelo de investigación.

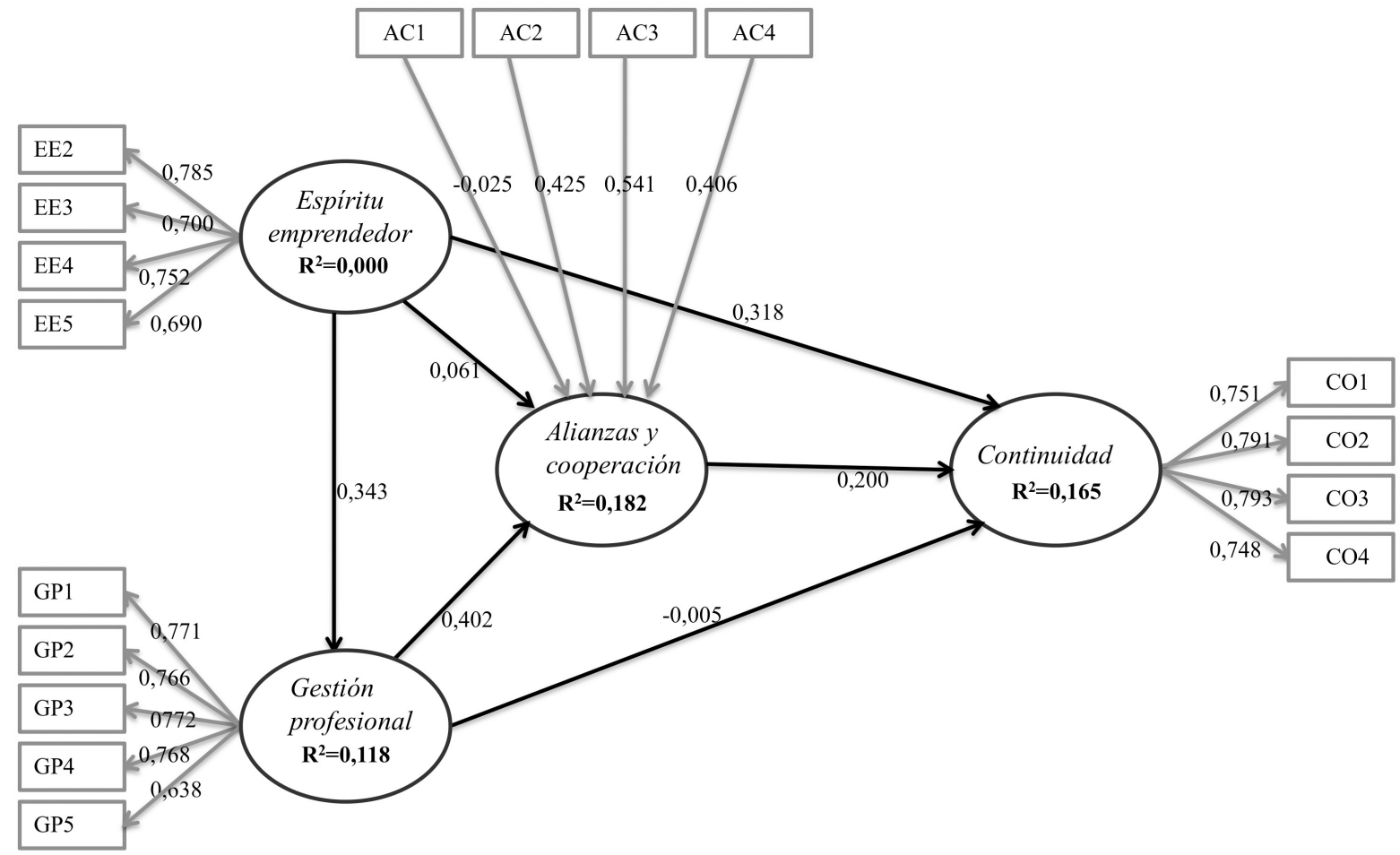

Fuente: elaboración propia

Bañegil Palacios, T.M., Barroso Martínez, A. y Tato Jiménez, J.L. (2011). Profesionalizarse, emprender y aliarse para que la empresa familiar continúe. Revista de Empresa Familiar, 1(2), 27-41. 
Partial Least Squares (PLS) que es una técnica de modelización de ecuaciones estructurales que utiliza un enfoque de estimación basado en componentes principales (Chin, 1998). El programa de software utilizado ha sido SmartPLS 2.0 (M3) Beta, Hamburg (Ringle, Wende, Will, 2005).

Nuestro modelo presenta medidas asociadas a los constructos de carácter reflectivo y de carácter formativos (Fornell, 1982). En PLS, los indicadores reflectivos están determinados por el constructo y, de ahí, que covaren al nivel de dicho constructo (Hulland, 1999). En el modelo tienen indicadores reflectivos los constructos Espíritu Emprendedor, Gestión Profesional de la Empresa Familiar y Continuidad de la Empresa Familiar. En cambio, una medida formativa implica que el constructo es expresado como una función de los ítems, esto es, los ítems observados forman o preceden el constructo. Es el caso de Alianzas y Cooperación.

La fiabilidad se relaciona con la coherencia de las medidas. Los ítems que miden un constructo altamente fiable están fuertemente correlacionados, indicando que todos ellos miden el mismo concepto. La fiabilidad del constructo mide la rigurosidad de los indicadores al medir las variables latentes (Chin, 1998), es decir, la consistencia interna de todos los indicadores al medir el concepto. Este tipo de fiabilidad puede medirse con el alfa de Cronbach o la fiabilidad compuesta (Werts, Linn, y Jöreskog, 1974).

En nuestro modelo, para el caso de los constructos con indicadores reflectivos, la fiabilidad compuesta podemos verla en la tabla 1. Los resultados hablan de la fiabilidad de nuestros constructos, ya que la cifra más baja es de 0,822167 del constructo Espíritu Emprendedor, que supera la fijada por la literatura como límite. En el caso del constructo medido mediante indicadores formativos (Alianzas y Cooperación), la fiabilidad de sus indicadores debe ser interpretada sobre la base de su contenido substantivo y en función de los pesos y no de las cargas (Chin, 1998). Es también necesario realizar un análisis de multicolinealidad. El estadístico más utilizado para estimar la multicolinealidad es el llamado factor de inflación de la varianza (FIV). Cuando el FIV es mayor o igual a 5 supone una situación problemática, y si es mayor o igual a 10 entonces se trata de un auténtico problema (Mathieson, Peacock y Chin, 2001). Nuestros valores son todos menores de 5 (el mayor es 1,519) por lo que no indican problemas de multicolinealidad, ni en el caso de los indicadores ni en el de la dimensiones.

\section{Tabla 1}

Evaluación del modelo de medida.

\begin{tabular}{cccc}
\hline & $\begin{array}{c}\text { Factor } \\
\text { de }\end{array}$ & & \\
$\begin{array}{c}\text { Constructo/Di } \\
\text { mensión/ }\end{array}$ & $\begin{array}{c}\text { Inflación } \\
\text { de la } \\
\text { Indicador }\end{array}$ & Peso & Carga \\
& $\begin{array}{c}\text { Varianz } \\
\text { a (VIF) }\end{array}$ & & \\
& & & \\
\hline
\end{tabular}

\begin{tabular}{lr}
\hline \multicolumn{2}{l}{ Espíritu Emprendedor (EE) } \\
(Constructo Latente Agregado de Primer Orden) \\
\multicolumn{2}{l}{ Fiabilidad Compuesta $\left(\mathrm{r}_{\mathrm{c}}\right)=0,822167$} \\
\multicolumn{2}{l}{ Varianza Extraída Media (AVE) $=0,536828$} \\
\hline EE1 & 0,555 \\
EE2 & 0,785 \\
EE3 & 0,700 \\
EE4 & 0,752 \\
EE5 & 0,690 \\
\hline
\end{tabular}

\section{Gestión Profesional}

(Constructo Latente de Primer Orden)

Fiabilidad Compuesta $\left(r_{c}\right)=0,860677$

Varianza Extraída Media $(\mathrm{AVE})=0,553972$

\begin{tabular}{ll}
\hline GP1 & 0,771 \\
GP2 & 0,766 \\
GP3 & 0,772 \\
GP4 & 0,769 \\
GP5 & 0,635 \\
GP6 & 0,611 \\
GP7 & 0,359 \\
\hline
\end{tabular}

Alianzas y Cooperación (CLF)

(Constructo Latente Agregado de Primer Orden)

Fiabilidad Compuesta $\left(r_{c}\right)=$ n.a. ${ }^{(1)}$

Varianza Extraída Media (AVE) $=$ n.a. ${ }^{(1)}$

\begin{tabular}{lll}
\hline AC1 & 1,246 & $-0,025$ \\
AC2 & 1,519 & 0,425 \\
AC3 & 1,405 & 0,541 \\
AC4 & 1,138 & 0,406 \\
\hline
\end{tabular}

\section{Continuidad}

(Constructo Latente de Primer Orden)

Fiabilidad Compuesta $\left(r_{c}\right)=0,854336$

Varianza Extraída Media $(\mathrm{AVE})=0,594716$

$\begin{array}{ll}\mathrm{CO} 1 & 0,751 \\ \mathrm{CO} 2 & 0,791 \\ \mathrm{CO} 3 & 0,793 \\ \mathrm{CO} 4 & 0,748\end{array}$

(1) n.a. No aplicable

Fuente: elaboración propia 
Una vez comprobada la no existencia de multicolinealidad entre los indicadores formativos del constructo, pasamos a observar los valores $\mathrm{t}$ obtenidos del procedimiento de remuestreo bootstrap (tabla 2). Todos los indicadores de Alianzas y Cooperación (tanto los significativos como los no significativos, son retenidos para estimar el modelo PLS. Las razones de tal hecho son, siguiendo la literatura (Diamantopoulos and Winklhofer, 2001; Bollen and Lennox, 1991; y Fornell et al., 1996): (1) que, en PLS, estas medidas no pueden ser observadas hasta una vez efectuado el análisis. (2) Además, ya que todas estas medidas están basadas en la literatura y en un análisis previo como fue el panel de expertos, es conceptualmente interesante saber qué facetas de las Alianzas y Cooperación son significativas y cuáles no.

Tabla 2

Valores $\mathrm{t}$.

\begin{tabular}{ccc}
\hline Indicador & Valor t & $\begin{array}{l}\text { Nivel de } \\
\text { significación }\end{array}$ \\
\hline AC1 & 0,163 & n.s. \\
AC2 & 1,915 & $*$ \\
AC3 & 2,357 & $* *$ \\
AC4 & 2,542 & $* *$ \\
\hline $\begin{array}{l}\text { (1) Nivel de significación para una t de student de 1 cola: Para } \mathrm{n}=500 \\
\text { submuestras: } \\
* * * \mathrm{p}<0,001 ; * * \mathrm{p}<0,01 ; * \mathrm{p}<0,05) \\
\mathrm{t}(0,001 ; 499)=3,106644601 ; \mathrm{t}(0,01 ; 499)=2,333843952 ; \mathrm{t}(0,05 ; 499) \\
=1,64791345\end{array}$
\end{tabular}

Fuente: elaboración propia

La validez convergente es analizada en PLS a través de la varianza extraída media (AVE) que proporciona la cantidad de varianza que un constructo obtiene de sus indicadores con relación a la cantidad de varianza debida al error de medida (Fornell y Larcker, 1981). En nuestro modelo tenemos cuatro constructos con indicadores reflectivos (Espíritu Emprendedor, Gestión Profesional de la E.F. y Continuidad) cuyos valores respectivos del AVE están representados en la tabla 1. Como puede comprobarse, todos ellos cumplen la regla de ser mayor a 0,5 .

La validez discriminante indica que un constructo es diferente a otro. Presentamos una tabla de correlaciones entre las puntuaciones (scores) del constructo y el resto de medidas (Fornell y Larcker, 1981).

Los resultados obtenidos pueden verse en la tabla 3. Todos los indicadores cargan más en su constructo que en el resto.

\section{Tabla 3}

Correlaciones entre puntuaciones de los constructos con indicadores reflectivos.

\begin{tabular}{llll}
\hline Indicador & Continuidad & $\begin{array}{l}\text { Espíritu } \\
\text { emprendedor }\end{array}$ & $\begin{array}{l}\text { Gestión } \\
\text { profesional }\end{array}$ \\
\hline CO1 & $\mathbf{0 , 7 5 1 4 9}$ & 0,315983 & 0,24513 \\
CO2 & $\mathbf{0 , 7 9 0 8 3 8}$ & 0,249748 & 0,11604 \\
CO3 & $\mathbf{0 , 7 9 3 4 6 4}$ & 0,317012 & 0,123854 \\
CO4 & $\mathbf{0 , 7 4 7 7 3 9}$ & 0,184776 & 0,059379 \\
\hline EE2 & 0,344642 & $\mathbf{0 , 7 8 4 6 9}$ & 0,222149 \\
EE3 & 0,225773 & $\mathbf{0 , 7 0 0 2 9 4}$ & 0,04525 \\
EE4 & 0,178754 & $\mathbf{0 , 7 5 1 7 8 7}$ & 0,342289 \\
EE5 & 0,273303 & $\mathbf{0 , 6 8 9 9 1 1}$ & 0,296073 \\
\hline GP1 & 0,16679 & 0,221917 & $\mathbf{0 , 7 7 0 9 9 1}$ \\
GP2 & 0,143178 & 0,219038 & $\mathbf{0 , 7 6 5 6 2}$ \\
GP3 & 0,071064 & 0,190768 & $\mathbf{0 , 7 7 1 5 6 1}$ \\
GP4 & 0,089883 & 0,187046 & $\mathbf{0 , 7 6 8 7 6 4}$ \\
GP5 & 0,207489 & 0,394428 & $\mathbf{0 , 6 3 4 7 8 8}$ \\
\hline
\end{tabular}

Fuente: elaboración propia

\subsection{Evaluación del modelo de investigación}

Los coeficientes $\beta$ representan los coeficientes path o pesos de regresión estandarizados. Indican la fuerza relativa de las relaciones estadísticas.

Para cada camino path o relación entre constructos los valores deseables deberían estar por encima de 0,3 o más altos, tomando 0,2 como límite mínimo (Chin, 1998a). En nuestro caso, y tal y como se desprende de las siguiente tabla, tan solo los paths del constructo Espíritu Emprendedor sobre Alianzas Estratégicas y Cooperación y el de Gestión Profesional sobre Continuidad no superan el límite mínimo. 
Tabla 4

Valores de los coeficientes $\beta$ o coeficientes path.

\begin{tabular}{llll}
\hline Variable & $\begin{array}{l}\text { Espíritu } \\
\text { Emprendedor }\end{array}$ & $\begin{array}{l}\text { Gestión } \\
\text { Profesio-- } \\
\text { nal }\end{array}$ & $\begin{array}{l}\text { Alianzas y } \\
\text { Coope- } \\
\text { ración }\end{array}$ \\
\hline $\begin{array}{l}\text { Gestión } \\
\text { Profesional }\end{array}$ & 3.979 & & \\
$\begin{array}{l}\text { Alianzas y } \\
\text { Cooperación }\end{array}$ & 0,061 & 3,139 & \\
Continuidad & 3,875 & $-0,005$ & 0,200 \\
\hline
\end{tabular}

Fuente: elaboración propia

Una medida del poder predictivo de un modelo es el valor $\mathrm{R}^{2}$ para las variables latentes dependientes. Falk y Millar (1992) señalan que la varianza explicada de las variables endógenas $\left(\mathrm{R}^{2}\right)$ debería ser mayor o igual a 0,1 . En nuestro modelo tenemos como variables dependientes: Gestión Profesional de la E.F., Alianzas y Cooperación y Continuidad. El valor de $\mathrm{R}^{2}$ para estos constructos es, respectivamente, de 0,118 ; 0,182 y de 0,165 , valores que superan las restricciones de estos autores, lo cual puede apreciarse en la figura 3 .

En nuestro análisis hemos incluido discusiones sobre la relevancia predictiva de los constructos dependientes mediante el test de Stone-Geisser que arroja el indicador $\mathrm{Q}^{2}$.

Según Sellin (1989) un valor superior a cero indica que la predictibilidad del modelo es relevante. Este test sigue un procedimiento de blindfolding donde se omiten parte de lo datos para un determinado constructo durante la estimación de parámetros para, a continuación, intentar estimar lo que se ha omitido usando los parámetros estimados (Chin, 1998). En nuestro caso pueden verse estos valores en la tabla 5 .

\section{Tabla 5}

Predictibilidad de los constructos Dependientes.

\begin{tabular}{lll}
\hline Constructo & $\begin{array}{l}\text { Q2 Stone- } \\
\text { Geisser }^{(\mathbf{1})}\end{array}$ & Predictibilidad \\
\hline $\begin{array}{l}\text { Gestión } \\
\text { Profesional }\end{array}$ & 0,059 & Sí \\
$\begin{array}{l}\text { Alianzas y } \\
\text { Cooperación }\end{array}$ & 0,073 & Sí \\
Continuidad & 0,073 & Sí
\end{tabular}

(1) Procedimiento Blindfolding. Cross Validadted Redundancy con distancia de omisión 7.

Fuente: elaboración propia

\subsection{Contraste de hipótesis planteadas}

Los resultados que ofrece nuestro modelo pueden observarse en la tabla 6 .

\section{Principales conclusiones y futuras líneas de investigación}

El primer resultado obtenido podría sorprender pues se rechaza la hipótesis que dice que la Gestión Profesional de las E.F. influye positivamente en la Continuidad de la E.F. $\left(\mathrm{H}_{1}\right)$. La explicación que podemos dar es que, en realidad, estamos midiendo la influencia en la continuidad de diferentes órganos de gobierno de la empresa, lo cuales muchas empresas familiares consideran poco útiles para su continuidad.

Otro resultado apreciable es que la formación de alianzas y cooperación influye en la continuidad del negocio $\left(\mathrm{H}_{2}\right)$. La intensidad es fuerte (valor del coeficiente path) aunque algunos autores señalan que ciertas características de la empresa familiar podrían afectar tanto a la elección del compañero para formar la alianza como a la forma jurídica de la misma, e incluso podría poner en peligro la continuidad de la empresa familiar. Así, Swinth y Vinton (1993) argumentan que las alianzas estratégicas entre empresas familiares tienen mayores probabilidades de éxito debido a que ambas comparten determinados objetivos, valores y convicciones. Esto podría llevar a plantearnos una futura línea de investigación donde analizásemos el efecto moderador de una variable que distinguiese entre diferentes tipos de socios con los que realizar alianzas para la continuidad de la empresa.

Un tercer resultado es que la Gestión Profesional de las E.F. influye positivamente en la formación de Alianzas y la Cooperación $\left(\mathrm{H}_{3}\right)$. Este resultado concuerda con la literatura revisada y además tiene un efecto importante. Creemos que determinado tipo de órganos de dirección y la actuación de profesionales ajenos a la familia pueden acrecentar la confianza de terceros a la hora de realizar alianzas y buscar cooperación. 


\section{Tabla 6}

Contraste de hipótesis planteadas.

\begin{tabular}{|c|c|c|c|c|}
\hline Hipótesis & $\begin{array}{l}\text { Efecto } \\
\text { sugerido }\end{array}$ & $\begin{array}{l}\text { Coeficientes } \\
\text { path } \\
\text { estandarizados }\end{array}$ & $\begin{array}{l}\text { Valor } t^{(1)} \\
\text { (Bootstrap) }\end{array}$ & $\begin{array}{l}\text { Hipótesis } \\
\text { Soportada }\end{array}$ \\
\hline $\begin{array}{l}\mathrm{H}_{1} \text { : La Gestión Profesional influye positivamente } \\
\text { en la Continuidad }\end{array}$ & + & $-0,005$ & 0,079 & n.s. \\
\hline $\begin{array}{l}\mathrm{H}_{2} \text { : La formación de Alianzas y la Cooperación } \\
\text { influye positivamente en la Continuidad }\end{array}$ & + & 0,2 & $1,969(*)$ & Sí \\
\hline $\begin{array}{l}\mathrm{H}_{3} \text { : La Gestión Profesional influye positivamente } \\
\text { en la formación de Alianzas y la Cooperación }\end{array}$ & + & 0,402 & $3,680(* * *)$ & Sí \\
\hline Cooperación & + & 0,061 & 0,722 & n.s. \\
\hline $\begin{array}{l}\mathrm{H}_{5}: \quad \text { El Espíritu Emprendedor influye } \\
\text { positivamente en la Continuidad }\end{array}$ & + & 0,318 & $3,984(* * *)$ & Sí \\
\hline $\begin{array}{llll}\mathrm{H}_{6}: \quad \text { El Espíritu } \quad \text { Emprendedor } & \text { influye } \\
\text { positivamente en la Gestión Profesional } & \end{array}$ & + & 0,343 & $3,811(* *)$ & Sí \\
\hline
\end{tabular}

(1)Nivel de significación para una t de student de 1 cola: Para $\mathrm{n}=500$ submuestras:

$* * * \mathrm{p}<0,001 ; * * \mathrm{p}<0,01 ; * \mathrm{p}<0,05)$

$\mathrm{t}(0,001 ; 499)=3,106644601 ; \mathrm{t}(0,01 ; 499)=2,333843952 ; \mathrm{t}(0,05 ; 499)=1,64791345$

Fuente: elaboración propia

Otra de las hipótesis no soportada es que el Espíritu Emprendedor influye en la formación de Alianzas y la Cooperación $\left(\mathrm{H}_{4}\right)$. Sin embargo, existe literatura que puede aclarar el porqué de tal hecho. Así, los investigadores Donckles y Fröhlich (1991) y Nordqvist, Habbershon y Melin (2008) señalan que las empresas familiares constituyen un contexto único para el espíritu emprendedor debido a las características específicas de las empresas familiares, es decir, estas empresas poseen un conjunto específico de recursos y capacidades que promueven o restringen las actividades emprendedoras.

Por tanto, dichas empresas poseen características que pueden fomentar el comportamiento emprendedor en la empresa a través de los continuos objetivos, las valiosas relaciones sociales, la supervivencia y la orientación a largo plazo; y también poseen características que pueden restringir dicho comportamiento como la mayor aversión al riesgo o la percepción diferente del entorno dependiendo del nivel generacional de la familia implicada (Kellermans, Eddleston, Barnett y Pearson (2008).
Este segundo conjunto de factores pueden ser la causa de nuestro resultado. Una posible forma de estudiarlo sería realizando un análisis multigrupo en función del grado de aversión al riesgo y la generación de la familia implicada, pues podemos encontrar literatura que señala que las empresas familiares tienen una mayor orientación emprendedora en la etapa del fundador, y este espíritu emprendedor va disminuyendo conforme las siguientes generaciones se van implicando en la empresa (Kellermans, Eddleston, Barnett y Pearson, 2008); sin embargo otros estudios muestran que las empresas familiares de segunda generación y multigeneracionales muestran un mayor comportamiento emprendedor que las empresas familiares de primera generación (Casillas, Moreno y Barbero, 2010), aunque si bien es cierto, a partir de la tercera generación el nivel de espíritu emprendedor comienza a disminuir.

Los resultados obtenidos indican además que el Espíritu Emprendedor influye positivamente en la Continuidad de la empresa familiar $\left(\mathrm{H}_{5}\right)$, y lo hace con bastante fuerza. Este resultado es acorde a la literatura. 
Por último, también se acepta la $\mathrm{H}_{6}$, donde el Espíritu Emprendedor influye positivamente en la Gestión Profesional de la E.F. Igualmente acorde con la literatura.

\section{Limitaciones}

A pesar de la robustez metodológica seguida y de los resultados del modelo reflejados en las páginas anteriores, existen ciertas limitaciones que queremos poner de manifiesto a la hora de realizar una valoración global de los resultados.

La primera limitación viene derivada de la metodología PLS, la cual no habla de causalidad sino de predictibilidad entre las variables independientes y la dependiente por tratarse de modelización flexible. En este sentido, hemos de reconocer que pueden existir distintos modelos alternativos que serán para nosotros objeto de futuras investigaciones.

Otra limitación que encontramos es la que hace referencia a las precauciones que debemos tener a la hora de generalizar los resultados. El estudio se ha desarrollado en el contexto de empresas extremeñas, por tanto, la generalización de estos resultados en el ámbito internacional deberá tener presentes los aspectos contingentes diferenciales.

\section{Bibliografía}

Aldrich, H. and Cliff, J. (2003). The pervasive effects of family on entrepreneurship: toward a family embeddedness perspective. Journal of Business Venturing, 18(5), 573-596.

Audretsch, D., Bönte, W. and Keilbach, M. (2008). Entrepreneurship capital and its impact on knowledge diffusion and economic performance. Journal of Business Venturing, 23(6), 687-698.

Blumentritt, T. P., Keyt, A. D. and Astrachan, J. H. (2007). Creating an environment for successful nonfamily CEOs: an exploratory study of good principals, Family Business Review, 20(4), 321-335.

Bollen, K. and Lennox, R. (1991). Conventional Wisdom on Measurement: A Structural
Equation Perspective. Psychological Bulletin, 110(2), 305-314.

Cabrera, K y Martín, J. (2010). La influencia de las relaciones intergeneracionales en la formación y el compromiso del sucesor: efectos sobre el proceso de sucesión de la empresa familiar. Revista Europea de Dirección y Economía de la Empresa, 19(2), 111-128.

Casillas, J.; Moreno, A.; Barbero, J. (2010). A configurational approach of the relationship between entrepreneurial orientation and growth of family firms. Family Business Review, 23(1), 27-44.

Cepeda Carrión, G. (2006). Understanding the link between knowledge management and firm performance: articulating and codifying critical knowledge areas. International Journal of Knowledge and Learning, 2(3-4), 238-262.

Chin, W.W. (1998a). The Partial Least Squares Approach to Structural Equation Modeling. En Marcoulides, G. A. (ed.) Modern Methods for Business Research. Fullerton: California State University.

Chin, W.W. (1998b). Issues and opinion on structural equation modeling, MIS Quarterly, 22(1), 7-16.

Chirico, F. (2008). Knowledge accumulation in family firms: evidence from four cases studies. International Small Business Journal, 26(4), 433-462.

Chittoor, R. and Das, R. (2007). Professionalization of management and succession performance: a vital linkage. Family Business Review, 20(1), 65-79.

Chu, W. (2011). Family ownership and firm performance: Influence of family management, family control, and firm size. Asia Pacific Journal of Management, 28(4), 833-851.

Claver, E., Rienda, L. y Pertusa, E. M. (2004). Un marco teórico para la continuidad de la empresa familiar desde un punto de vista estratégico. Esic market, 118, 229-259.

Claver, E., Rienda, L. y Quer, D. (2008). Factores familiares y compromiso internacional: Evidencia empírica en las empresas españolas. 
Cuadernos de Economía y Dirección de la Empresa, 35, 7-26.

Cole, P. (2000). Understanding family business relationships: preserving the family in the business. Family Journal, 8(4), 351-359.

Diamantopoulos, A. and Winkholfer, H.M. (2001). Index construction with Formative Indicators: An Alternative to Scale Development. Journal of Marketing Research, 38(2), 269-277.

Donckels, R. and Lambrecht, J. (1999). The Reemergence of Family-Based Enterprises in East Central Europe: What Can Be Learned from Family Business Research in the Western World? Family Business Review, 12(2), 171191.

Dyck, B., Mauws, M., Starke, F.A. and Mischke, G.A. (2002). Passing the baton. The importance of sequence, timing, technique and communication in executive succession. Journal of Business Venturing, 17(2), 143-162.

Falk, R.F. and Millar, N.B. (1992), A primer for soft modelling. Ohio: University of Akron.

Fernández, E. (1996). Innovación, tecnología y alianzas estratégicas. Madrid: Civitas.

Fernández, N. y Bringmann, E. (2007). El impacto de la cultura organizacional y del liderazgo en las empresas familiares, pp. 34283444. En Ayala, J. L. (coord.), Conocimiento, Innovación y Emprendedores: camino al futuro. Logroño: Universidad de la Rioja.

Fernández, Z. and Nieto, M.J. (2005). Internationalization strategy of small and medium-sized family businesses: Some influential factors. Family Business Review, 18(1), 77-89.

Fornell, C. (1982). A Second Generation of Multivariate analysis. New York: Praeger.

Fornell, C., Johnson, M. D., Anderson, E.W., Cha, J. and Bryant, B. E. (1996). The american customer satisfaction index: nature, purposes. and findings. Journal of Marketing, 60(4), 7-18.

Fornell, C. and Larcker, D.F. (1981). Evaluating Structural Equation Modelswith Unobservable
Variables and Measurement Error, Journal of Marketing Research, 18(1), 39-50.

Fuentes, G. y Fernández, R. (2008). Alianzas estratégicas en la internacionalización de la empresa. En Casillas Bueno, J. C. (coord.), Internacionalización de la Empresa Familiar. Sevilla: Edición Digital @ tres.

Fuentes, G., Vallejo, M. C. y Fernández, R. (2011). Explorando las alianzas en la internacionalización de bodegas familiares españolas, argentinas y chilenas. Revista de Empresa Familiar, 1(1), 69-81.

Gallo, M. A. (1998). La sucesión en la empresa familiar. Colección Estudios e Informes de la Caixa, 12.

Gallo, M. A. y Amat, J. M. (2003): Los secretos de las empresas familiares centenarias. Barcelona: Ediciones Deusto.

Gallo, M. A., Ariño, A., Máñez, I. and Cappuyns, K. (2004). Internationalization via strategic alliances in family businesses. Working paper IESE Business School, 540.

Galve, C. (2002). Propiedad y Gobierno: la empresa familiar. Ekonomiaz: Revista vasca de economía, 50, 158-181.

Giovannoni, E., Maraghini, M. P. and Riccaboni, A (2011). Transmitting knowledge across generation: the role of management accounting practices. Family Business Review, 24(2), 126-150.

Gortázar, C. (2007). Principales aspectos jurídicos y societarios del protocolo familiar. En Amat, J. M. y Corona, J.F. (coord.), El protocolo familiar. La experiencia de una década (pp. 195-214). Barcelona: Ediciones Deusto.

Hall, A., Melin, L. and Nordqvist, M. (2001). Entrepreneurship as radical change in the family business: exploring the role of cultural patterns. Family Business Review, 14(3), 193-208.

Hall, A. and Nordqvist, M. (2008). Professional management in family business: toward an extended understanding. Family Business Review, 21(1), 51-69. 
Handler, W. C. (1994). Succession in Family Business: A Review of the Research. Family Business Review, 7(2), 133-157.

Hulland, J. (1999). Use of Partial Least Squares (PLS) in Strategic Management Research: A Review of Four Recent Studies. Strategic Management Journal, 20, 195-204.

Ibrahim, A. B., Soufami, K., Poutziouris, P. and Lam, J. (2004). Qualities of an effective successor: the role of education and training. Education + Training, 46(8/9), 474-480.

James JR., H.S. (1999). What can the family contribute to business? Examining contractual relationships. Family Business Review, 12(1), 61-72.

Jaskiewicz, P. and Klein, S. (2007). The impact of goal alignment on board composition and board size in family businesses. Journal of Business Research, 60, 1080-1089.

Kellermanns, F. W; Eddleston, K. A (2004). Feuding Families: When Conflict Does a Family Firm Good. Entrepreneurship, Theory and Practice, 28(3), 209-228.

Lansberg, I. (2007). El protocolo familiar dentro del contexto cultural español. En Amat, J. M. y Corona, J.F. (coord.), El protocolo familiar. La experiencia de una década (pp. 27-36). Barcelona: Ediciones Deusto.

Leach, P. (2002): La Empresa Familiar. Barcelona: Ediciones Granica.

Le Breton-Miller, I., Miller, D. and Steier, L. P. (2004). Toward an integrative model of effective FOB succession. Entrepreneurship Theory and Practice, 28(4), 305-328.

Lozano, M. (2003). Expansión de empresas familiares mediante alianzas internacionales. Revista de Empresa, 4, 98-108.

Mathieson, K., Peacock, E. and Chin, W. W. (2001). Extending the technology acceptance model: the influence of perceived user resources. The DATA BASE for Advances in Information Systems, 32(3), 86-112.

Martínez, J. (2007). Introducción al protocolo familiar y al consejo de familia. En Amat, J. M. y Corona, J.F. (coord.) El protocolo familiar. La experiencia de una década (pp 61-70). Barcelona: Ediciones Deusto.

McCann, J. E., Leon-Guerrero, A. and Haley, J. D. (2001). Strategic goals and practices of innovative family businesses. Journal of Small Business Management, 39(1), pp. 50-59.

Miller, N. J.; McLeod, H.; Oh, K. Y. (2001). Managing family businesses in small communities. Journal of Small Business Management, 39(1), pp. 73-87.

Naldi, L., Nordqvist, M. Sjöberg, K. and Wiklund, J. (2007). Entrepreneurial Orientation, Risk Taking and Performance in Family Firms. Family Business Review, 23, 33-47.

Nunnally, J. (1978). Psychometric Theory. New York: McGraw-Hill.

Ordóñez de Pablos, P. (2001) la Gestión del Conocimiento en los RR.HH. Oviedo: Tesis Doctoral. Universidad de Oviedo.

Pistrui, D., Huang, W., Oksoy, D., Jing, Z. and Welsch, H. (2001). Entrepreneurship in China: characteristics, attributes and family forces shaping the emerging private sector. Family Business Review, 14(2), 141-152.

Podsakoff, N.P., Shen, W. y Podsakoff, P.M. (2006). The role of formative measurement models in strategic management research: review, critique, and implications for future research. Research Methodology in Strategy and Management, 3, 197-252.

Ringle, C.M., Wende, S. and Will, S. (2005), SmartPLS 2.0 (M3) Beta, Hamburg: http://www.smartpls.de

Roessl, D. (2005). Family Businesses and Interfirm Cooperation. Family Business Review, 18(3), 203- 214.

Royer, S.; Simons, R.; Boyd, B.; Rafferty, A. (2008). Promoting Family: A Contingency Model of Family Business Succession. Family Business Review, 21(1), 15-30.

Sánchez-Crespo, A. J. (2009): El Protocolo Familiar. Una aproximación práctica a su preparación y ejecución. Madrid: SánchezCrespo Abogados y Consultores 
Sánchez-Crespo, A. J., Bellver, A. y Sánchez, A. M. (2005). La empresa familiar: manual para empresarios. Barcelona: Ediciones Deusto.

Sellin, N. (1989). Partial Least Square Modeling in Research of Educational Achievement. In Wilfred Boss and Rainer H. Lehmannn (Eds,), Reflections on Educational Achievement: Papers in Honour of T. Neville Postlethwaite (pp. 256-267). New York: Waxmann Munster.

Shane, S. and Venkataraman, S (2000). The Promise of Entrepreneurship as a Field of Research. Academy of Management Review, 25(1), 217-226.

Swinth, R. and Vinton, K. (1993). Do family owned businesses have a strategic advantage in international joint ventures? Family Business Review, 6(1), 19-30.

Tagiuri, R. and Davis, J. A. (1996). Bivalent attributtes of the family firm. Family Business Review, 9(2), 199-208.
Ulhøi, J. (2005). The social dimensions of entrepreneurship. Technovation, 25(8), 939-946.

Ulubasoglu, A. M., Akdis, M. and Kök, S. B. (2009). Internationalization and Alliance Formation: Evidence from Turkish SMEs. International Small Business Journal, 27(3), 337-361.

Van Gils, A. and Zwart, P. S. (2009). Alliance Formation Motives in SMEs: An Explorative Conjoint Analysis Study. International Small Business Journal, 27(1), 5-37.

Werts, C.E., Linn, R.L. and Jöreskog, K.G. (1974). Interclass Reliability Estimates: Testing Structural Assumptions. Educational and Psychological Measurement, 34(1), 25-33. 